\title{
Increased gut hormones and insulin sensitivity index following a 3-d intervention with a barley kernel-based product: a randomised cross-over study in healthy middle-aged subjects
}

\author{
Anne C. Nilsson*, Elin V. Johansson-Boll and Inger M. E. Björck \\ Food for Health Science Centre, Lund University Specialized Centres, Lund University, Lund SE-221 OO, Sweden \\ (Submitted 5 March 2015 - Final revision received 20 May 2015 - Accepted 9 June 2015 - First published online 11 August 2015)
}

\begin{abstract}
Certain purified indigestible carbohydrates such as inulin have been shown to stimulate gut-derived hormones involved in glycaemic regulation and appetite regulation, and to counteract systemic inflammation through a gut microbiota-mediated mechanism. Less is known about the properties of indigestible carbohydrates intrinsic to food. The aim of this study was to investigate the possibility to affect release of endogenous gut hormones and ameliorate appetite control and glycaemic control by ingestion of a whole-grain cereal food product rich in NSP and resistant starch in healthy humans. In all, twenty middle-aged subjects were provided with a barley kernel-based bread (BB) or a reference white wheat bread during 3 consecutive days, respectively, in a randomised cross-over design study. At a standardised breakfast the following day (day 4), blood was collected for the analysis of blood (b) glucose regulation, gastrointestinal hormones, markers of inflammation and markers of colonic fermentation; $3 \mathrm{~d}$ of intervention with BB increased gut hormones in plasma (p) the next morning at fasting (p-glucagon-like peptide-1; 56\%) and postprandially (p-glucagon-like peptide-2; $13 \%$ and p-peptide YY; $18 \%$ ). Breath $\mathrm{H}_{2}$ excretion and fasting serum (s) SCFA concentrations were increased (363 and $18 \%$, respectively), and b-glucose (22\%) and s-insulin responses (17\%) were

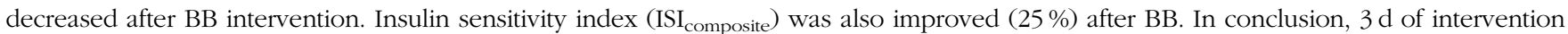
with BB increased systemic levels of gut hormones involved in appetite regulation, metabolic control and maintenance of gut barrier function, as well as improved markers of glucose homoeostasis in middle-aged subjects, altogether relevant for the prevention of obesity and the metabolic syndrome.
\end{abstract}

Key words: Glucose tolerance: Dietary fibre: Colonic fermentation: Barley: Gut hormones: Appetite regulation: Dietary prevention

Epidemiological studies have shown that diets rich in whole grain may protect against CVD, type 2 diabetes and certain types of cancer ${ }^{(1,2)}$. Recent prospective studies also indicate a lowered total mortality and CVD mortality ${ }^{(3)}$. Despite the accumulating epidemiological evidence, the mechanisms involved in the protective effects of whole grains remain unclear, and controlled intervention studies indicate variable effects of whole-grain $v$. refined-grain diets ${ }^{(4)}$. Consequently, there is a need to elucidate the causal relationship between dietary fibre (DF) characteristics and health outcome. In addition to the DF per se, the phytonutrients associated with DF may also contribute to this factor. Further, the structural features and degree of processing - for example, grinding - may affect the resistant starch (RS) contents that add to the total DF complex present in whole-grain food.

Whole-grain diets have been suggested to ameliorate blood (b) glucose control $^{(4)}$. Several mechanisms have been put forward - for example, acute effects, by reducing the rate of upper-gut digestion and absorption of starch, or semi-acute and longer-term effects, originating from gut fermentation of DF. In this respect, SCFA, formed during gut fermentation, have been proposed to be involved ${ }^{(5,6)}$.

Gut-derived hormones play an important role in energy regulation and are proposed to be involved as modulators of inflammation as well. Glucagon-like peptide (GLP)-1 and peptide YY (PYY) participate in the regulation of appetite and glucose metabolism $^{(7-9)}$. The gut hormone GLP-2 appears to be involved as a regulator of gut barrier function ${ }^{(10)}$, and it has been suggested that inflammatory processes linked to diminished gut barrier function with concomitant systemic influx of lipopolysaccharides (LPS) may promote development of obesity and diabetes ${ }^{(11)}$. GLP-1, PYY and GLP-2 are secreted from L-cells present throughout the intestinal epithelium with highest density towards the distal ileum and colon and are

Abbreviations: BB, barley kernel-based bread; DF, dietary fibre; GLP, glucagon-like peptide; iAUC, incremental AUC; iPeak, incremental peak; OXM, oxyntomodulin; PYY, peptide YY; RS, resistant starch; WWB, white wheat bread.

* Corresponding author: A. C. Nilsson, fax +46 4622245 32, email anne.nilsson@food-health-science.lu.se 
co-released in the presence of carbohydrates ${ }^{(7,12)}$. Studies are at hand implicating that certain gut substrates may affect L-cells and stimulate gut hormones through a mechanism linked to gut fermentation. SCFA receptors co-localised with enteroendocrine L-cells appear to provide a link between fermentation and stimulation of gut hormones, and SCFA receptor deficiency impairs SCFA-induced gut hormone secretion both in vitro and in vivo ${ }^{(13)}$. In rats, dietary supplementation with oligofructose increased the number of L-cells in the proximal colon, as well as the circulating GLP-1 concentration, compared with a control diet $^{(14)}$. Interestingly, a 2 -week dietary supplementation with oligofructose-enriched inulin increased p-GLP-1 and PYY concentrations in healthy adults ${ }^{(15)}$. Further, intrinsic DF present in evening meals of barley kernels or brown beans facilitated b-glucose regulation and beneficially affected gut hormones such as GLP-1 or PYY as well as subjective appetite at a subsequent breakfast meal in young healthy subjects ${ }^{(16-18)}$. The important role of gut hormones in systemic metabolism and inflammation suggests that an increased endogenous release of these hormones would be of importance with respect to prevention of cardiometabolic disorders and obesity.

The purpose of the present study was to investigate the role of indigestible carbohydrates (NSP and RS) present in barley kernel-based bread (BB) on metabolism and gut hormones in a cohort of healthy, middle-aged subjects. The intervention with $\mathrm{BB}$ or white wheat bread (WWB) reference was performed for 3 consecutive days before the overnight study, where a standardised breakfast was provided on day 4 and blood samples were collected in the postprandial phase (0-150 min). Blood parameters included measures of glucose metabolism (glucose and insulin) and a range of gut-derived hormones (GLP-1, GLP-2, PYY, oxyntomodulin (OXM)), ghrelin, inflammatory markers (IL-6, IL-18 and adiponectin) and NEFA. In addition, s-SCFA was determined, and subjective appetite sensations were registered. Breath $\mathrm{H}_{2}$ was measured as an indicator of gut fermentative activity.

\section{Methods}

\section{Test subjects}

A total of twenty healthy volunteers, three men and seventeen women, aged 64.1 (sD 5.9) years, with normal BMI (mean 23.6 $\left(\mathrm{sD} 2.3 \mathrm{~kg} / \mathrm{m}^{2}\right)$ ) participated in this study. Recruitments of test subjects were conducted through advertisements in local newspapers during September 2010 and January 2011. The experimental study was conducted at Lund University, and was completed in March 2011. The inclusion criteria were as follows: men and women between 50 and 70 years of age, normal to slightly overweight (BMI $19-28 \mathrm{~kg} / \mathrm{m}^{2}$ ), fasting plasma glucose $\leq 6.1 \mathrm{mmol} / \mathrm{l}$, non-smoker, overall healthy and with no known metabolic disorders or food allergies. Subjects who took anti-hypertensive medications (one subject) and prescription-free painkillers without anti-inflammatory action were also included. All the enrolled test subjects completed the study. This study was conducted according to the guidelines laid down in the Declaration of Helsinki, and all the procedures involving human subjects were approved by the Regional
Ethical Review Board in Lund, Sweden (Reference 2010/457). Written informed consent was obtained from all the subjects. The study was registered at ClinicalTrials.gov (NCT01718431).

\section{Experimental procedure}

The test subjects participated in two 3-d dietary interventions, with either test products or reference products taken in a random order separated by at least 2 weeks (between 2.0 and 4.5 weeks, mean 2.7 (SEM 0.8) weeks). A test product consisting of $\mathrm{BB}$ and a reference product consisting of WWB were included.

Intervention protocol. The quantity of the test products and reference products to be ingested daily during the 3-d study period was standardised to provide $100 \mathrm{~g}$ of potentially available starch per day, calculated as the difference between total starch and RS. This corresponded to $9 \cdot 1 \mathrm{~g}$ total DF/d (72\% NSP and $28 \%$ RS) in the case of the reference WWB intervention and $37.6 \mathrm{~g}$ total $\mathrm{DF} / \mathrm{d}(55.8 \% \mathrm{NSP}$ and $45.2 \% \mathrm{RS})$ in the case of the $\mathrm{BB}$ test intervention period. The daily intake of test bread or reference bread was distributed into three equal portions to be consumed at approximately 08.00, 14.00 and 21.00 hours for the first $2 \mathrm{~d}$. On day 3, half of the daily intake ( $50 \mathrm{~g}$ available starch) was distributed equally between the 08.00 and 14.00 hours meals, and the other half ( $50 \mathrm{~g}$ available starch) was consumed at 21.00 hours in the evening.

The subjects were encouraged to standardise their meal pattern and to avoid alcohol, excessive physical exercise or foods rich in DF during the 3-d intervention periods. Furthermore, they should not have taken antibiotics or probiotics during the previous 2 weeks and also throughout the study period. After the evening meal (consisting of the last portion of the test product or reference product, respectively), on day 3 , the subjects were fasting until the standardised breakfast was served at the experimental department. The subjects arrived at the experimental department at 07.30 hours. An intravenous cannula (BD Venflon; Becton Dickinson) was inserted into an antecubital vein to be used for blood sampling. Fasting blood samples were collected and subjective appetite and breath $\mathrm{H}_{2}$ were registered before providing the standardised breakfast. The standardised breakfast (see below) was provided at approximately 08.00 hours and was ingested within $13 \mathrm{~min}$. During the $2.5 \mathrm{~h}$ of repeated sampling, the subjects were told to maintain low physical activity.

Standardised breakfast on day 4. The standardised breakfast consisted of $122.9 \mathrm{~g}$ WWB corresponding to $50 \mathrm{~g}$ of available carbohydrates, analysed according to Holm et al. ${ }^{(19)}$, and $2.5 \mathrm{dl}$ tap water.

Recipes and preparation of reference and test products as well as the standardised breakfast white wheat bread (white wheat bread: reference product and standardised breakfast). The WwB was baked according to a standardised procedure in a home baking machine (Tefal Home Bread Model no. 573102; menu choice, programme 2 (white bread, 
Colonic fermentation and gut hormones

Table 1. Portion size and carbohydrate composition of the reference and test products, respectively*

\begin{tabular}{|c|c|c|c|c|c|c|c|}
\hline \multirow[b]{2}{*}{ Meals } & \multirow[b]{2}{*}{ Portion size } & \multicolumn{3}{|c|}{ Starch } & \multicolumn{2}{|c|}{ NSP } & \multirow[b]{2}{*}{ Total DF } \\
\hline & & Total & Available & RS & Insoluble & Soluble & \\
\hline \multicolumn{8}{|l|}{$g / d$} \\
\hline WWB & 233.8 & 103 & 100 & $2 \cdot 5$ & $4 \cdot 7$ & 1.8 & $9 \cdot 1$ \\
\hline BB & 338.2 & 117 & 100 & $17 \cdot 0$ & $14 \cdot 0$ & $6 \cdot 6$ & 37.6 \\
\hline \multicolumn{8}{|l|}{$\%$ DM } \\
\hline WWB & - & $77 \cdot 4$ & $75 \cdot 5$ & 1.92 & 3.6 & 1.4 & $6 \cdot 9$ \\
\hline BB & - & $74 \cdot 3$ & 63.5 & $10 \cdot 8$ & $8 \cdot 8$ & $4 \cdot 2$ & $23 \cdot 8$ \\
\hline
\end{tabular}

RS, resistant starch; DF, dietary fibre; WWB, white wheat bread; BB, barley kernel-based bread.

${ }^{*}$ Available starch is calculated as the difference between total starch ${ }^{(20)}$ and $\mathrm{RS}^{(21)}$. Values of total starch are based on means of two replicates, RS means of six replicates and NSP are based on means of three replicates. Included in total DF are RS and insoluble and soluble NSP, determined gravimetrically according to Asp et al. ${ }^{(22)}$.

$1000 \mathrm{~g}$, quick (time $2 \cdot 32 \mathrm{~h}$ ))). The bread was made from $540 \mathrm{~g}$ of white wheat flour (Kungsörnen AB), $360 \mathrm{~g}$ water, $4.8 \mathrm{~g}$ dry yeast and $4.8 \mathrm{~g} \mathrm{NaCl}$ (without iodine). After cooling, the bread was sliced and wrapped in aluminium foil in portion sizes, placed in plastic bags and stored in a freezer $\left(-20^{\circ} \mathrm{C}\right)$. The crust was removed from the WWB intended for the standardised breakfast.

Barley kernel-based bread (test product). A total of $595 \mathrm{~g}$ barley kernels (Finax) were boiled in $520 \mathrm{~g}$ water for $12 \mathrm{~min}$ and then cooled for $30 \mathrm{~min}$ at ambient room temperature. Water was completely absorbed into the kernels when cooked. Added to the kernels were $105 \mathrm{~g}$ wheat flour, $6 \mathrm{~g}$ dry yeast, $5 \mathrm{~g}$ salt and $300 \mathrm{~g}$ water. The dough was kneaded for $4 \mathrm{~min}$ (Electrolux AKM 3000, N23 N25) and proofed for $30 \mathrm{~min}$ in a bowl, followed by another proofing $(35 \mathrm{~min}$ ) in a baking tin. The baking tin was covered with aluminium foil and baked in a household oven at $225^{\circ} \mathrm{C}$ until the inner temperature of the bread reached $96^{\circ} \mathrm{C}$ with a pan of water present to maximise steam. After baking, the bread was cooled at ambient room temperature, removed from the tin and wrapped in wet towels. After cooling, the towels were removed and the bread samples were placed in plastic bags at room temperature overnight. The day after, the bread samples were sliced and wrapped in aluminum foil in portion sizes, placed in plastic bags and stored in a freezer $\left(-20^{\circ} \mathrm{C}\right)$. On days before consumption, the test persons were instructed to take a daily portion of bread from the freezer and thaw it at ambient temperature, with the bread still wrapped in aluminum foil and maintained in the plastic bag.

\section{Chemical analysis of the test and reference products}

The test and reference products were analysed with respect to total starch ${ }^{(20)}, \mathrm{RS}^{(21)}$ and insoluble and soluble $\mathrm{NSP}^{(22)}$. Before analysis of total starch and NSP, the bread samples were airdried and milled. RS was analysed on products as eaten. Available starch was calculated by subtracting RS from total starch. The composition of the test and reference products is presented in Table 1.

\section{Sampling and analysis of physiological variables}

Venous blood samples were taken to determine serum (s-) insulin, s-NEFA, s-SCFA, s-IL-6, s-IL-18 and s-adiponectin and for the determination of plasma (p-) GLP-1, p-GLP-2, p-PYY, p-OXM and p-ghrelin. Finger-prick capillary blood samples were taken for the determination of whole b-glucose concentrations (HemoCue ${ }^{\circledR}$ B-glucose; HemoCue AB). Breath $\mathrm{H}_{2}$ was measured as an indicator of colonic fermentation using a Gastro + (Bedfont EC60 Gastrolyzer; Bedfont). Measures of subjective appetite sensations (satiety, hunger and desire to eat) were obtained using a 100-mm visual analogue scale. Glucose, $\mathrm{H}_{2}$ and appetite sensations were determined at fasting and at 15, 30, 45, 60, 90, 120 and $150 \mathrm{~min}$ after commencing the standardised breakfast. Insulin level was determined at similar time points, with the exception of $15 \mathrm{~min}$. GLP-1 was analysed at fasting and at 30, 45, 60, 90 and $150 \mathrm{~min}$, and GLP-2 and ghrelin were analysed at fasting and at 30, 60, 90 and $150 \mathrm{~min}$. IL-6, IL-18, OXM and PYY were determined at fasting and at 60 and $120 \mathrm{~min}$ after start of the breakfast. Adiponectin level was analysed at fasting and at $120 \mathrm{~min}$, and NEFA was determined at fasting and at $150 \mathrm{~min}$. SCFA was determined at fasting.

Serum and plasma were separated by centrifugation and immediately stored in a freezer $\left(-40^{\circ} \mathrm{C}\right)$ until analysis. Bloodcollecting tubes intended for the analysis of p-ghrelin, p-GLP-1, p-GLP-2, p-PYY and p-OXM were prepared using an inhibition cocktail consisting of a dipeptidyl peptidase-4 (DPPIV) inhibitor $\left(10 \mu \mathrm{l} / \mathrm{ml}\right.$ blood) (Millipore) and Trasylol ${ }^{\circledR} 10000 \mathrm{KIE} / \mathrm{ml}$ aprotinin $(50 \mu \mathrm{l} / \mathrm{ml}$ blood) (Bayer HealthCare AG) before blood sampling. Tubes containing the inhibition cocktail were kept on ice until use for a maximum of $6 \mathrm{~d}$. Plasma for the analysis of ghrelin was additionally treated with $1 \mathrm{~m}-\mathrm{HCl}$ before freezing (10:1).

Serum insulin levels were measured using an enzyme immunoassay kit procedure (Mercodia) and performed on an integrated immunoassay analyser (CODA Open Microplate System; Bio-Rad Laboratories). Commercial kits based on ELISA were used for the determination of s-adiponectin (Mercodia Adiponectin ELISA; Mercodia), p-OXM (Cusabio Biotech), p-PYY (3-36 and 1-36), p-GLP-2 (Yanaihara Institute Inc.), p-GLP-1 (active 7-36) (Alpco Diagnostics), p-ghrelin (BioVendor GmbH), s-IL-6 (R\&D Systems) and s-IL-18 (MBL). Serum NEFA levels were measured using an enzymatic colorimetric method (NEFA C, ACS-ACOD method; Wako Chemicals $\mathrm{GmbH}$ ), and s-SCFA (acetate, propionate and butyrate) were determined using a GC method $^{(23)}$.

\section{Calculations and statistical methods}

Statistical evaluations of glucose and insulin areas and peaks were based on incremental changes from fasting concentrations. Calculations regarding all other test variables were performed 
using actual values. The incremental AUC (iAUC) and AUC were calculated for each subject and the respective breakfast meal, using the trapezoid model. GraphPad Prism (version 5; GraphPad Software) was used for graph plotting and calculation of areas. Incremental peak (iPeak) concentrations were determined for glucose and insulin as individual maximum postprandial increase from baseline. Individual mean values during the experimental day were calculated for physiological test variables when a postprandial response after breakfast was scarcely seen. Total SCFA were calculated as the sum of the concentrations of acetate, propionate and butyrate. As a measure of insulin resistance, a homoeostatic model assessment $($ HOMA-IR) was used (fasting glucose $(\mathrm{mmol} / \mathrm{l}) \times$ fasting insulin $(\mathrm{mU} / \mathrm{l}) / 22 \cdot 5)^{(24)}$. For the assessment of insulin sensitivity, a modified composite insulin sensitivity index (ISI composite $_{\text {), also }}$ called the Matsuda index, was used, including measures of b-glucose and s-insulin both at fasting and in the postprandial phase after the standardised breakfast (ISI composite $: 10000 / \sqrt{\text { fasting glucose }(\mathrm{mg} / \mathrm{dl}) \times \text { fasting insulin }}$ $(\mu \mathrm{U} / \mathrm{ml}) \times$ mean glucose concentrations $0-120 \mathrm{~min}(\mathrm{mg} / \mathrm{dl}) \times$ mean insulin concentrations $0-120 \min (\mu \mathrm{U} / \mathrm{ml}))^{(25,26)}$. The determination of the Matsuda indices was modified with respect to analysing postprandial concentrations for insulin and glucose after consuming standardised WWB based on $50 \mathrm{~g}$ rapidly available starch instead of $75 \mathrm{~g}$ glucose.

Significant differences in test variables after the test and reference products were assessed with ANOVA (general linear model) using Minitab Statistical Software (release 14-16, Minitab; Minitab Inc.). In the cases of unevenly distributed residuals (tested using the Anderson-Darling test and considered unevenly distributed when $P<0 \cdot 05$ ), Box-Cox transformations were performed on the data before ANOVA. Differences between the products at different time points were evaluated using a mixed model (PROC MIXED in SAS release 9.2 and 9.3; SAS Institute Inc.) with repeated measures and an autoregressive covariance structure. Due to previous suggestions that SCFA may be involved in the release of gut hormones, relationships between total SCFA and gut hormones (PYY, GLP-1 and GLP-2) were investigated using Pearson's correlation in Minitab Statistical Software (release 14-16). Randomisation of the order in which the test or reference products were taken and power calculations were performed in Minitab Statistical Software (release 14-16). If a value from a test subject was missing for one of the products, the test subject was excluded from that specific calculation. Values of $P \leq 0.05$ were considered significant. Data are expressed as mean values with their standard errors of the mean; $n 20$.

\section{Power calculation}

The primary outcome measure for power calculations was change in b-glucose iAUC 0-120 min after the standardised breakfast. Number of test subjects required for the study was determined in Minitab, using previous results of 'overnight' effects of barley kernel meals on glucose iAUC 0-120 min $^{(16)}$. Assuming a difference of $70 \mathrm{mmol} / \mathrm{l}$ per min between the WWB and the BB intervention, and an SD of $82 \mathrm{mmol} / \mathrm{l}$ per min, with $\alpha=0.05$ and $1-\beta=0.8$, about thirteen to seventeen test subjects were required (two-tailed test). We decided to increase the number of test subjects to twenty due to the lack of information from previous similar studies regarding the cohort included in the present study (middle-aged adults).

\section{Results}

Gastrointestinal hormones (peptide $Y Y$, glucagon-like peptide-1, glucagon-like peptide-2, oxyntomodulin and ghrelin)

A main effect of the test products was observed on p-PYY concentrations in the morning following the 3-d intervention, revealing higher levels following BB compared with WWB $(P=0.042)$. When calculating a mean of p-PYY for the time period 0-150 min, increased concentrations were seen after BB (18\%; $P=0.043)$ compared with WWB (Table 2). Consumption of $\mathrm{BB}$ for $3 \mathrm{~d}$ resulted in significantly increased concentrations of p-GLP-1 at fasting on day $4(56 \% ; P=0.006)$. The mean p-GLP-2 concentrations were increased during the standardised breakfast on day 4 (13\%; $P=0.042)$ after BB intake for $3 \mathrm{~d}$, as compared with WWB,

Table 2. Gut hormone responses following $3 \mathrm{~d}$ of intervention with reference or test products, respectively (Mean values with their standard errors)

\begin{tabular}{|c|c|c|c|c|c|}
\hline \multirow[b]{2}{*}{ Test variables } & \multicolumn{2}{|c|}{ WWB } & \multicolumn{2}{|c|}{ BB } & \multirow[b]{2}{*}{$\% \dagger$} \\
\hline & Mean & SEM & Mean & SEM & \\
\hline $\mathrm{p}$-GLP-1, fasting value (pmol/l, $n$ 19) & 1.0 & 0.4 & 1.6 & 0.5 & $+56^{\star \star}$ \\
\hline p-GLP-1, mean 0-150 $\min (\mathrm{pmol} / \mathrm{l}, n$ 19) & 1.8 & 0.4 & 1.8 & 0.4 & +5 \\
\hline $\mathrm{p}-\mathrm{GLP}-2$, fasting value $(\mu \mathrm{g} / \mathrm{l}, n$ 19) & $2 \cdot 7$ & 0.3 & 2.9 & 0.3 & $+9 \ddagger$ \\
\hline p-GLP-2, mean 0-150 $\min (\mu \mathrm{g} / \mathrm{l}, n 19)$ & $3 \cdot 1$ & 0.4 & 3.5 & 0.5 & $+13^{*}$ \\
\hline p-PYY, fasting value ( $\mu \mathrm{g} / \mathrm{l}, n$ 17) & 0.76 & 0.13 & 0.84 & $0 \cdot 16$ & +10 \\
\hline $\mathrm{p}-\mathrm{PYY}$, mean $0-120 \mathrm{~min}(\mu \mathrm{g} / \mathrm{l}, n$ 17) & 0.71 & 0.13 & 0.84 & 0.14 & $+18^{*}$ \\
\hline p-Ghrelin, fasting value (ng/l, $n 20)$ & $55 \cdot 8$ & $7 \cdot 1$ & $58 \cdot 6$ & $8 \cdot 6$ & +6 \\
\hline $\mathrm{p}$-Ghrelin, mean 0-150 $\mathrm{min}$ (ng/l, $n$ 20) & $66 \cdot 7$ & $10 \cdot 0$ & $69 \cdot 2$ & $11 \cdot 8$ & +4 \\
\hline $\mathrm{p}$-OXM, fasting value $(\mu \mathrm{g} / \mathrm{l}, n \mathrm{16})$ & 1.4 & 0.6 & 1.2 & 0.5 & -16 \\
\hline p-OXM, mean 0-120 min ( $\mathrm{ug} / \mathrm{l}, n$ 16) & 1.5 & 0.6 & 1.4 & 0.6 & -5 \\
\hline
\end{tabular}

WWB, white wheat bread; BB, barley kernel-based bread; p, plasma; GLP-1 and 2, glucagon-like peptides 1 and 2; PYY, peptide YY; OXM, oxyntomodulin.

Significantly different from WWB: * $P<0.05,{ }^{\star *} P<0.01$ (ANOVA; general linear model, Minitab).

† Percentage of differences in concentrations of test variables after BB compared with WWB.

$\ddagger P=0.06$. 
and a tendency $(P=0 \cdot 06)$ towards increased fasting concentrations of p-GLP-2 was observed after BB (Table 2).

No significant differences were observed in the concentrations of $\mathrm{p}$-ghrelin or $\mathrm{p}$-OXM following the standardised breakfast, depending on intervention product (Table 2).

\section{Breath hydrogen and serum SCFA}

Inclusion of the BB test product resulted in increased breath $\mathrm{H}_{2}$ levels at fasting on day 4 compared with the WWB (146\%; $P=0.007)$. The increased levels of $\mathrm{H}_{2}$ were maintained during the standardised breakfast (363\%; $P=0 \cdot 000)$ (Fig. 1; Table 3). Intake of $\mathrm{BB}$ for $3 \mathrm{~d}$ increased fasting concentrations of s-acetate and total s-SCFA $(18 \% ; P=0.013$ and $16 \% ; P=0.012$, respectively, Table 3$)$. A tendency $(P=0 \cdot 1)$ towards an increase in s-butyrate levels was observed after BB compared with WWB.

\section{Blood glucose and serum insulin}

B-glucose and s-insulin responses at the standardised breakfast are presented in Fig. 2. In comparison with the intervention with WWB, the 3-d intervention with BB beneficially affected

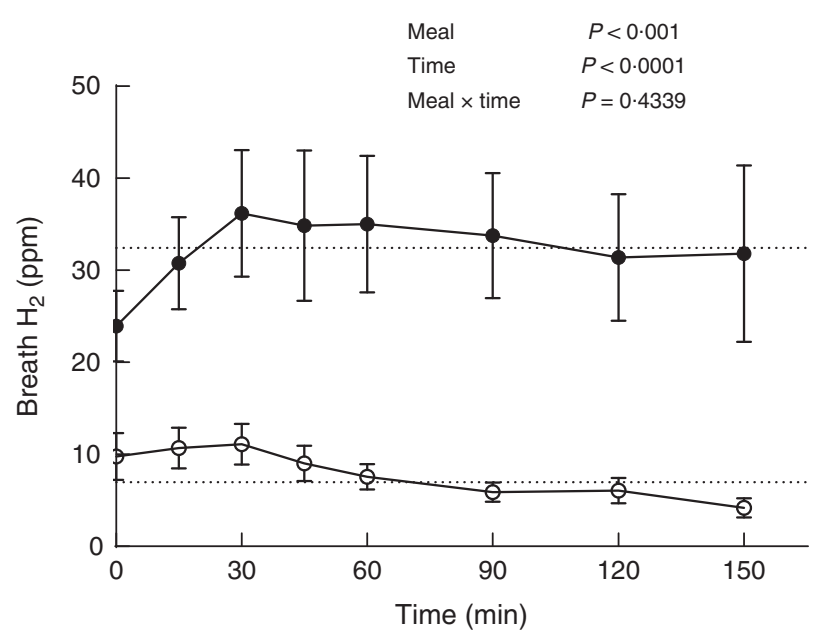

Fig. 1. Breath hydrogen excretion during the experimental day. Mean postprandial breath hydrogen concentrations at a standardised breakfast following $3 \mathrm{~d}$ of intervention with barley kernel-based bread (BB) or white wheat bread (WWB), respectively. Dotted lines at the $y$-axis indicate mean values for BB (32.5 (SEM 6.77) parts per million (ppm)) and WWB (7.01 (SEM 1.18) ppm). Values are means with their standard errors; $n 20$ (repeated measures; mixed model in SAS). WWB; $\longrightarrow$ - $\mathrm{BB}$ the b-glucose response at the following standardised breakfast in terms of lower iPeak (BB and WWB: $2 \cdot 8$ (SEM 0.2) and 3.4 (SEM $0 \cdot 2) \mathrm{mmol} / \mathrm{l}$, respectively, $-17 \% ; P=0.014)$ and lower iAUC 0-120 min (BB and WWB: 152 (SEM 12) and 194 (SEM 18) mmol/1 per $\mathrm{h}$, respectively, $-22 \% ; P=0 \cdot 018)$. In addition, in comparison with WWB, BB lowered the s-insulin iPeak (BB and WWB: $0 \cdot 21$ (SEM 0.02) and 0.24 (SEM 0.02) nmol/l per min, respectively, $-11 \% ; P=0.024)$ and s-insulin iAUC 0-120 min (BB and WWB:
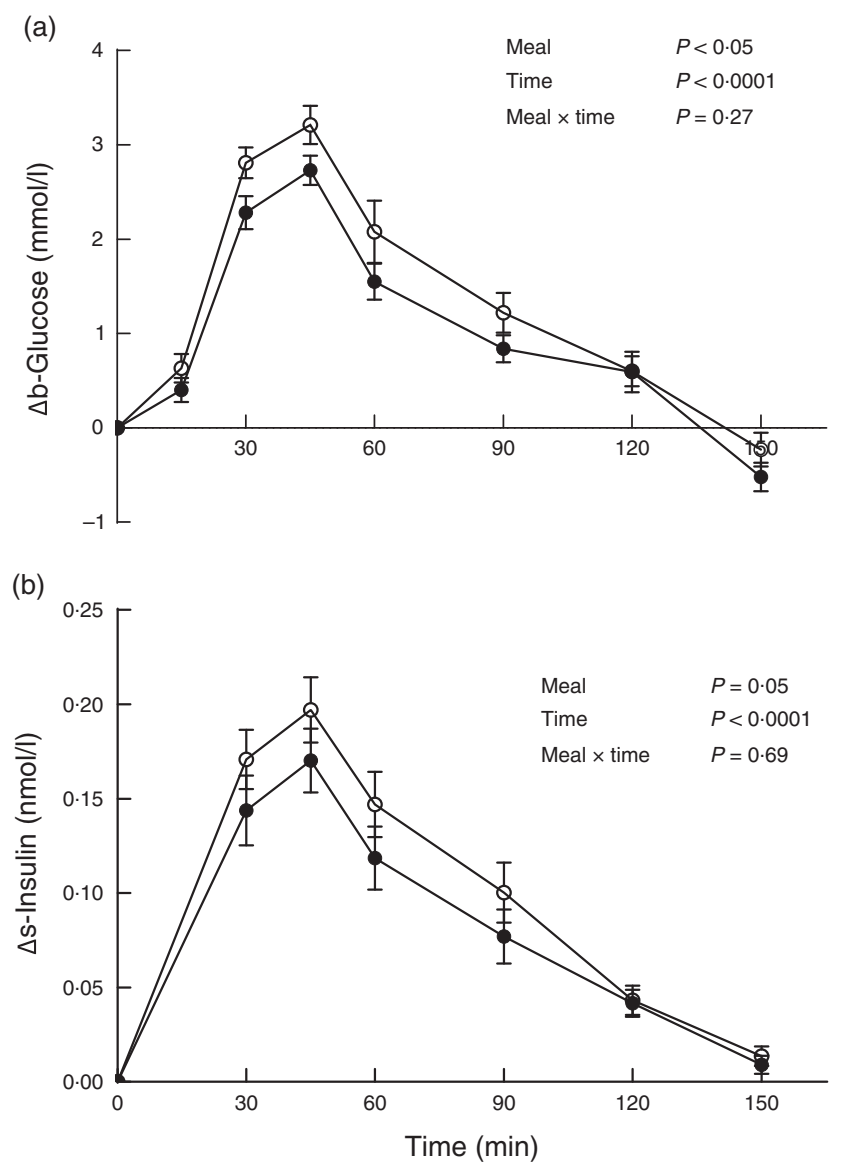

Fig. 2 Incremental blood (b)-glucose and serum (s)-insulin responses during the experimental day. Incremental b-glucose (a) and s-insulin (b) response at a standardised breakfast following $3 \mathrm{~d}$ of intervention with barley kernel-based bread (BB) or white wheat bread (WWB), respectively. Values are means with their standard errors; $n 20$ (repeated measures; mixed model in SAS). - - WWB; $\longrightarrow$ B, BB.

Table 3. Serum (s)-SCFA and breath hydrogen following $3 \mathrm{~d}$ of intervention with reference or test products, respectively (Mean values with their standard errors)

\begin{tabular}{|c|c|c|c|c|c|}
\hline \multirow[b]{2}{*}{ Test variables } & \multicolumn{2}{|c|}{ WWB } & \multicolumn{2}{|c|}{ BB } & \multirow[b]{2}{*}{$\% \dagger$} \\
\hline & Mean & SEM & Mean & SEM & \\
\hline Breath $\mathrm{H}_{2}$, fasting value (ppm, $n$ 20) & $9 \cdot 8$ & $2 \cdot 6$ & $24 \cdot 0$ & 3.9 & $+146^{\star \star}$ \\
\hline Breath $\mathrm{H}_{2}$, mean 0-150 min (ppm, $n$ 20) & $7 \cdot 0$ & $1 \cdot 2$ & 32.5 & $6 \cdot 8$ & $+363^{\star \star \star}$ \\
\hline $\mathrm{s}$-Acetate, fasting value $(\mu \mathrm{mol} / \mathrm{l}, n 19)$ & 145 & 12 & 172 & 10 & $+18^{*}$ \\
\hline s-Propionate, fasting value ( $\mu \mathrm{mol} / \mathrm{l}, n$ 19) & $9 \cdot 8$ & 0.9 & $10 \cdot 5$ & 0.8 & +6 \\
\hline s-Butyrate, fasting value ( $\mu \mathrm{mol} / \mathrm{l}, n$ 18) & $14 \cdot 2$ & 0.9 & $16 \cdot 0$ & 0.7 & $+13 \ddagger$ \\
\hline Total s-SCFA, fasting value $(\mu \mathrm{mol} / \mathrm{l}, n 18)$ & 163 & 11 & 192 & 9 & $+18^{\star}$ \\
\hline
\end{tabular}

WWB, white wheat bread; BB, barley kernel-based bread; ppm, parts per million.

Significantly different from WWB: ${ }^{\star} P<0.05,{ }^{\star \star} P<0.01,{ }^{\star \star \star} P<0.001$ (ANOVA; general linear model, Minitab).

$\dagger$ Percentage of differences in concentrations of test variables after BB compared with WWB.

$\ddagger P=0.1$. 
Table 4. Whole blood (b)-glucose and serum (s)-insulin responses including measures of insulin resistance and insulin sensitivity at a standardised breakfast following $3 \mathrm{~d}$ of intervention with reference or test products, respectively

(Mean values with their standard errors)

\begin{tabular}{|c|c|c|c|c|c|}
\hline \multirow[b]{2}{*}{ Test variables } & \multicolumn{2}{|c|}{ WWB } & \multicolumn{2}{|c|}{ BB } & \multirow[b]{2}{*}{$\% \dagger$} \\
\hline & Mean & SEM & Mean & SEM & \\
\hline b-Glucose, fasting (mmol/l, $n$ 20) & $5 \cdot 5$ & 0.1 & $5 \cdot 6$ & 0.1 & +1 \\
\hline s-Insulin, fasting (nmol/l, n 20) & 0.029 & 0.003 & 0.028 & 0.003 & -3 \\
\hline HOMA-IR ( $n$ 20) & $1 \cdot 17$ & 0.12 & $1 \cdot 17$ & 0.15 & 0 \\
\hline ISI composite $_{(n \text { 20) }}$ & $12 \cdot 5$ & $1 \cdot 1$ & $14 \cdot 2$ & $1 \cdot 3$ & $+14^{* *}$ \\
\hline
\end{tabular}

WWB, white wheat bread; BB, barley kernel-based bread; HOMA-IR, homoeostatic model assessment insulin resistance; $I_{\text {composite, }}$ insulin sensitivity index.

** Significantly different from WWB $(P<0.01$; ANOVA; general linear model, Minitab).

$\dagger$ Percentage of differences in concentrations of test variables after BB Swap the $\dagger$ and ${ }^{* *}$ footnotes.

11.4 (SEM 1.2) and 13.8 (SEM) $1.3 \mathrm{nmol} / 1$ per min, respectively,

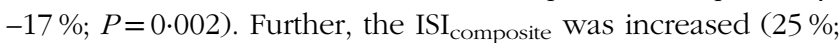
$P=0.005)$, indicating a higher insulin sensitivity on day 4 after $3 \mathrm{~d}$ of $\mathrm{BB}$ intervention compared with WWB. No effects were observed for fasting insulin resistance as estimated using HOMA-IR. Table 4 displays the results regarding insulin sensitivity variables and fasting glucose and insulin concentrations.

\section{Subjective appetite sensation, serum NEFA and markers of inflammation (serum-IL-6, serum-IL-18 and serum adiponectin)}

No significant differences in appetite sensations (satiety, hunger and desire to eat) were observed at the standardised breakfast meal depending on previous interventions (data not shown). However, a tendency for a main effect appeared for desire to eat ( $P=0.08$, repeated measures; mixed model in SAS), with less desire to eat in the case of the 3-d intervention period with BB.

No differences were observed in s-NEFA, s-IL-6, s-IL-18 or s-adiponectin levels depending on the intervention product $(P>0 \cdot 05$, data not shown).

\section{Relationships between markers of gut fermentation and gut hormones}

In the morning on day 4 in both the intervention periods, the fasting concentrations of total s-SCFA were positively associated with fasting concentrations of p-PYY $(r 0.51, P=0.038$ and $r 0.57, P=0.01$, after WWB and BB, respectively). Fasting concentrations of s-SCFA were similarly associated with postprandial p-PYY concentrations (mean $0-150 \mathrm{~min} ; r 0.51, P=0.032$ and $r 0.55, P=0.014$, after WNB and $\mathrm{BB}$, respectively). In addition, fasting s-SCFA after BB intervention was positively associated with postprandial (mean 0-150 min) concentrations of p-GLP-2 $(r 0 \cdot 53, P=0 \cdot 020)$.

\section{Discussion}

The present study investigated the impact of indigestible carbohydrates (NSP and RS) present in BB on endogenous release of gut hormones, metabolic risk markers and perceived appetite in healthy, normal-to-slightly overweight middle-aged subjects. We showed that intake of BB for $3 \mathrm{~d}$ markedly increased gut fermentation activity, manifested as increased breath $\mathrm{H}_{2}$ excretion and s-SCFA concentrations at a standardised breakfast on day 4, as compared with intake of the WWB reference. Further, circulating concentrations of gut hormones p-GLP-1, p-GLP-2 and p-PYY were significantly elevated following consumption of $\mathrm{BB}$ for $3 \mathrm{~d}$. In parallel, the present study demonstrates not only a lowered b-glucose response (iPeak and iAUC 0-120 min) at the standardised breakfast challenge after BB in comparison with the WWB, but also a reduced insulin response (iPeak and iAUC 0-120 min). Although no specific analysis of insulin sensitivity was performed using, for example, the clamp technique, the present study indicates improved insulin sensitivity as determined by an increased ISI $_{\text {composite }}$ after the $\mathrm{BB}$ intervention. The results are in accordance with studies showing improved insulin sensitivity in healthy subjects after a meal tolerance test in the morning, following intake of $60 \mathrm{~g} \mathrm{DF}$ from Hi-maize over a period of $24 \mathrm{~h}$ the previous day ${ }^{(27)}$. The authors suggested a mechanism related to increased rate of colonic fermentation. Similarly, insulin resistance, assessed using the euglycemic-hyperinsulinemic clamp technique, was improved in healthy subjects after 4 weeks intervention with Hi-maize RS (Hi-maize 260; $30 \mathrm{~g} / \mathrm{d}$; National Starch and Chemical) $^{(28)}$.

The underlying mechanisms are, however, not clarified, and other authors have, for example, argued that the beneficial effects of DF on insulin resistance are due to the interference of DF with the digestion and/or absorption of dietary proteins in the small intestine ${ }^{(29)}$. Consequently, effects of DF on insulin sensitivity was previously investigated in obese subjects with the metabolic syndrome after 18 weeks of DF supplementation and compared with a diet high in proteins, or a diet rich in both DF and proteins, and a control diet neither enriched in proteins nor in $\mathrm{DF}^{(29)}$. Insulin sensitivity was assessed using the euglycemic-hyperinsulinemic clamp technique. The results showed no improvement on insulin sensitivity after DF supplementation compared with the control diet. However, the high-protein diet impaired insulin sensitivity, resulting in significantly better insulin sensitivity after DF supplementation in comparison with the high-protein diet. In addition, when DF was added to the high-protein diet, the deleterious effect on insulin resistance was diminished. The DF supplement source in the obese study population $^{(29)}$ was composed of DF isolates consisting of nonsoluble and poorly fermentable DF from oat husk, where the $\beta$-glucans were removed. This is in contrast with the test product included in the present study, which instead was rich in highly fermentable soluble DF made up mostly of $\beta$-glucans. The results, thus, suggest that several mechanisms may be 
involved in eliciting beneficial effects and that different sources of DF may target different metabolic pathways.

In humans, supplementation with different DF has shown different effects on second meal overnight GLP-1 concentrations. Consequently, in healthy subjects, 4-week supplementation with RS $(30 \mathrm{~g} / \mathrm{d})$ did not affect GLP-1 concentrations at fasting or postprandially at breakfast the following day ${ }^{(28)}$, whereas an increase in GLP-1 was observed after 2 weeks of prebiotic supplementation containing oligofructose-enriched inulin (16 g/ d) ${ }^{(15)}$. Further, 24-h supplementation with a mixture of insoluble DF from oat extracts (depleted from $\beta$-glucans), wheat DF extracts and RS (Hi-maize 1043; National Starch and Chemical) did not increase GLP-1 concentrations the following day at fasting or following a standardised breakfast ${ }^{(30)}$. The differences in outcome on GLP-1 with different DF sources is not known but could be related to the differences in capacity to stimulate specific gut bacteria. The incretin GLP-1 has been ascribed both antidiabetic and anti-obesity features, and during the last few years there has been an increased interest regarding the possibility to stimulate endogenous GLP-1 release as a means to improve postprandial glucose metabolism ${ }^{(31,32)}$. In rats, dietary supplementation with oligofructose increased the number of L-cells in the proximal colon with a concomitant increase in endogenous GLP-1 concentrations ${ }^{(14)}$. In addition, specific key differentiation factors, involved in endocrine cell maturation, were up-regulated after oligofructose treatment. In this context, it was suggested that the promotion of L-cell development was possibly mediated through SCFA formation ${ }^{(14,28)}$. It can be speculated that the increase in circulating gut hormones observed in the present study may be a result from a shift in the microbial flora and an increased number of L-cells after BB intervention. However, the intervention time in the present study was $3 \mathrm{~d}$, and the regeneration time for enteroendocrine cells is usually $5-7 \mathrm{~d}^{(33)}$. Thus, probably additional mechanisms may be operative in the shorter time perspective of the present work, possibly involving increased activation of already-existing L-cells, and increased SCFA receptor signalling ${ }^{(34)}$. Tolhurst et al. ${ }^{(6)}$ suggested that the colonic L-cells may be directly influenced by SCFA (acetate and propionate), with a concomitant stimulation of GLP-1 and PYY release. Accordingly, rectal administration of acetate in hyperinsulinaemic overweight subjects increased circulating GLP-1 and PYY $1 \mathrm{~h}$ after administration ${ }^{(35)}$. The possibility to activate the L-cells by such a mechanism is a plausible hypothesis behind the results presented in this study, supported by an increased level of circulating s-SCFA (total of acetate, propionate and butyrate) and increased concentrations of gut hormones p-GLP-1, p-PYY and p-GLP-2 - all co-secreted from the L-cells ${ }^{(12)}$. The observed positive relationships between s-SCFA and p-PYY or p-GLP-2, respectively, further strengthen the possibility for such a mechanism. The results regarding effects of the specific indigestible carbohydrates present in the barley kernel product on release of gut hormones in a 11- to 14-h perspective, and the relationships between the gut hormones and colonic fermentation metabolites - that is, SCFA - are novel. However, studies regarding mechanisms of gut flora-mediated benefits on host metabolism are scarce and more studies are needed comparing efficacy of different DF as presented in foods, focusing on the relationships between gut fermentation and cardiometabolic benefits. A tentative mechanism for the metabolic benefits seen in the present study relates to the activation of specific gut bacteria by the intrinsic DF substrates in BB, causing stimulation of L-cells and/or generation of SCFA. Consequently, ingestion of $\mathrm{BB}$ for $3 \mathrm{~d}$ increased abundance of specific gut microbes in faeces, providing evidence for a mechanism related to gut fermentation (P Kovatcheva-Datchary, A Nilsson, R Akrami, Y Shiuan Lee, F De Vadder, T Arora, A Hallen, E Martens, I Björck and $\mathrm{F}$ Bäckhed, unpublished results).

This study is to our knowledge among the first to observe a simultaneous increase in several gut hormones in humans in response to intrinsic DF in a composite food. As a particularly interesting observation, there was an increase in p-GLP-2 concentrations after the intervention with BB. To our knowledge, only one report is available showing a similar increase in endogenous GLP-2 following intake of brown beans using a similar experimental design ${ }^{(18)}$. GLP-2 appears to be involved in epithelial cell proliferation and intestinal growth, and its importance for gut barrier functions has been increasingly acknowledged $^{(36)}$. LPS, released by unfavourable intestinal bacteria and absorbed into the systemic circulation, may promote a state of metabolic endotoxemia, and act to trigger subclinical inflammation and development of insulin resistance ${ }^{(37)}$, and even obesity ${ }^{(38)}$. Therefore, the gut epithelium provides an important protective barrier against translocation of endotoxins $^{(39)}$. Interestingly, prebiotic supplementation increased circulating concentrations of GLP-2, as well as decreased intestinal permeability and concentrations of $\mathrm{p}$-LPS in obese mice, altogether implicating a GLP-2-dependent mechanism ${ }^{(10)}$.

The present study has certain limitations. For example, instead of using the golden standard method to determine insulin resistance - that is, the hyperinsulinemic-euglycemic clamp - HOMA-IR, and the Matsuda index were used to estimate insulin sensitivity. Matsuda index has been shown to correlate well ( $r 0.73, P<0 \cdot 0001)$ with the rate of whole-body glucose disposal during the euglycemic insulin clamp ${ }^{(25)}$. In our study, we used a modified method for calculations of the Matsuda index. Typically, the postprandial determinations of glucose and insulin concentrations are performed after a $75 \mathrm{~g}$ glucose load. We instead assessed the Matsuda index after a standardised WWB breakfast, yielding $50 \mathrm{~g}$ available carbohydrates from starch. As a potential additional study limitation it should be noted that the relationship between s-SCFA and p-GLP-2 was obtained between fasting concentrations of p-SCFA and mean concentrations of p-GLP-2 during the intervention period ( $0-150 \mathrm{~min}$ ), and it cannot be ruled out that the discrepancy in the time may have affected the results. Regarding test subjects, an unbalanced sex distribution was present, providing only three male compared with seventeen female subjects. We did not investigate all the data only for women; however, re-calculating glucose and insulin data without men did not affect the outcome. Another possible study limitation was that the study design (food products consumed at home) did not allow for controlling compliance. However, the apparent difference in breath $\mathrm{H}_{2}$ at day 4 between test $\mathrm{BB}$ and reference WWB intervention indicates that the test subjects followed instructions regarding the test products. Finally, several response variables were investigated, and we cannot 
rule out that some of the detected associations were due to chance. However, we did not adjust for multiple testing as several of the investigated response variables were correlated; such adjustment would, therefore, be unduly conservative.

\section{Conclusions}

The present study demonstrates the metabolic benefits of ingestion of $\mathrm{BB}$ for $3 \mathrm{~d}$ in healthy middle-aged subjects, as manifested by increased concentrations of GLP-1, PYY and GLP-2, facilitated b-glucose regulation and improved insulin sensitivity. In addition, positive relationships were observed between total SCFA and gut hormones PYY and GLP-2, indicating a causal relationship between SCFA production and gut hormone secretion. These new findings suggest antiinflammatory, anti-diabetic and anti-obesogenic potential of foods rich in intrinsic indigestible carbohydrates (NSP/RS approximately $13 / 11 \%, \mathrm{DM})$, as present in the BB. It is suggested that the mechanisms are related to gut fermentation of the DF fraction in BB. These findings are novel and encouraging, and it is put forward that such foods may add to the preventive value of a healthy diet. However, more studies are needed to clarify the complex mechanisms underlying the relationships between colonic fermentation of certain DF and cardiometabolic benefits

\section{Acknowledgements}

This study was funded by the Lund University Antidiabetic Food Centre, a VINNOVA VINN Excellence Centre (grant no. 2013/46).

The authors' contributions were as follows: E. V. J.-B., I. M. E. B. and A. C. N. designed the study; E. V. J.-B. and A. C. N. conducted the study; E. V. J.-B. and A. C. N. analysed the data; E. V. J.-B, I. M. E. B. and A. C. N. evaluated the results and wrote the paper; and A. C. N. and I. M. E. B. had primary responsibility for the final content. All the authors read and approved the final manuscript.

The authors declare that they have no conflicts of interest.

\section{References}

1. von Ruesten A, Feller S, Bergmann MM, et al. (2013) Diet and risk of chronic diseases: results from the first 8 years of followup in the EPIC-Potsdam study. Eur J Clin Nutr 67, 412-419.

2. Grooms KN, Ommerborn MJ, Pham DQ, et al. (2013) Dietary fiber intake and cardiometabolic risks among US adults, NHANES 1999-2010. Am J Med 126, 1059-1067 (e1-4).

3. Wu H, Flint AJ, Qi Q, et al. (2015) Association between dietary whole grain intake and risk of mortality: two large prospective studies in US men and women. JAMA Intern Med 175, 373-384.

4. Harris Jackson K, West SG, Vanden Heuvel JP, et al. (2014) Effects of whole and refined grains in a weight-loss diet on markers of metabolic syndrome in individuals with increased waist circumference: a randomized controlled-feeding trial. Am J Clin Nutr 100, 577-586.

5. Cani PD, Everard A \& Duparc T (2013) Gut microbiota, enteroendocrine functions and metabolism. Curr Opin Pharmacol 13, 935-940.

6. Tolhurst G, Heffron H, Lam YS, et al. (2012) Short-chain fatty acids stimulate glucagon-like peptide-1 secretion via the G-protein-coupled receptor FFAR2. Diabetes 61, 364-371.
7. Sam AH, Troke RC, Tan TM, et al. (2012) The role of the gut/ brain axis in modulating food intake. Neuropharmacology $\mathbf{6 3}$, $46-56$.

8. Holst JJ (2007) The physiology of glucagon-like peptide 1. Physiol Rev 87, 1409-1439.

9. Persaud SJ \& Bewick GA (2014) Peptide YY: more than just an appetite regulator. Diabetologia 57, 1762-1769.

10. Cani PD, Possemiers S, Van de Wiele T et al. (2009) Changes in gut microbiota control inflammation in obese mice through a mechanism involving GLP-2-driven improvement of gut permeability. Gut 58, 1091-1103.

11. Delzenne NM, Neyrinck AM \& Cani PD (2013) Gut microbiota and metabolic disorders: how prebiotic can work? Br J Nutr 109, Suppl. 2, S81-S85.

12. Schwartz TW \& Holst B (2010) An enteroendocrine full package solution. Cell Metab 11, 445-447.

13. Psichas A, Sleeth ML, Murphy KG, et al. (2014) The short chain fatty acid propionate stimulates GLP-1 and PYY secretion via free fatty acid receptor 2 in rodents. Int J Obes (Lond) 39, 424-429.

14. Cani PD, Hoste S, Guiot Y, et al. (2007) Dietary non-digestible carbohydrates promote L-cell differentiation in the proximal colon of rats. Br J Nutr 98, 32-37.

15. Cani PD, Lecourt E, Dewulf EM, et al. (2009) Gut microbiota fermentation of prebiotics increases satietogenic and incretin gut peptide production with consequences for appetite sensation and glucose response after a meal. Am J Clin Nutr $\mathbf{9 0}$, $1236-1243$

16. Nilsson AC, Östman EM, Holst JJ, et al. (2008) Including indigestible carbohydrates in the evening meal of healthy subjects improves glucose tolerance, lowers inflammatory markers, and increases satiety after a subsequent standardized breakfast. J Nutr 138, 732-739.

17. Johansson EV, Nilsson AC, Ostman EM, et al. (2013) Effects of indigestible carbohydrates in barley on glucose metabolism, appetite and voluntary food intake over $16 \mathrm{~h}$ in healthy adults. Nutr J 12, 46.

18. Nilsson A, Johansson E, Ekstrom L, et al. (2013) Effects of a brown beans evening meal on metabolic risk markers and appetite regulating hormones at a subsequent standardized breakfast: a randomized cross-over study. PLOS ONE 8, e59985.

19. Holm J, Björck IME, Drews A, et al. (1986) A rapid method for the analysis of starch. Starch/Stärke 38, 224-226.

20. Björck IME \& Siljeström MA (1992) In-vivo and in-vitro digestibility of starch in autoclaved pea and potato products. J Sci Food Agric 58, 541-553.

21. Åkerberg AK, Liljeberg HG, Granfeldt YE, et al. (1998) An in vitro method, based on chewing, to predict resistant starch content in foods allows parallel determination of potentially available starch and dietary fiber. J Nutr 128, 651-660.

22. Asp N-G, Johansson C-G, Hallmer H, et al. (1983) Rapid enzymatic assay of insoluble and soluble dietary fiber. J Agric Food Chem 31, 476-482.

23. Brighenti F (1998) Summary of the conclusion of the working group on profibre interlaboratory study on determination of short chain fatty acids in blood. In Functional Properties of Non-Digestible Carbohydrates, pp. 150-153 [F Gullion, R Amadò, MT Amaral-Collaco, H Andersson, NG Asp, KEB Knudsen, M Champ, J Mathers, JA Robertson, I Rowland and JV Loo, editors]. Brussels: European Commission, DG XII, Science, Research and Development.

24. Matthews DR, Hosker JP, Rudenski AS, et al. (1985) Homeostasis model assessment: insulin resistance and beta-cell function from fasting plasma glucose and insulin concentrations in man. Diabetologia 28, 412-419.

25. Matsuda M \& DeFronzo RA (1999) Insulin sensitivity indices obtained from oral glucose tolerance testing: comparison 
with the euglycemic insulin clamp. Diabetes Care 22, 1462-1470.

26. DeFronzo RA \& Matsuda M (2010) Reduced time points to calculate the composite index. Diabetes Care 33, e93.

27. Robertson MD, Currie JM, Morgan LM, et al. (2003) Prior shortterm consumption of resistant starch enhances postprandial insulin sensitivity in healthy subjects. Diabetologia 46, 659-665.

28. Robertson MD, Bickerton AS, Dennis AL, et al. (2005) Insulinsensitizing effects of dietary resistant starch and effects on skeletal muscle and adipose tissue metabolism. Am J Clin Nutr 82, 559-567.

29. Weickert MO, Roden M, Isken F, et al. (2011) Effects of supplemented isoenergetic diets differing in cereal fiber and protein content on insulin sensitivity in overweight humans. Am J Clin Nutr 94, 459-471.

30. Weickert MO, Mohlig M, Koebnick C, et al. (2005) Impact of cereal fibre on glucose-regulating factors. Diabetologia $\mathbf{4 8}$, $2343-2353$.

31. Mansour A, Hosseini S, Larijani B, et al. (2013) Nutrients related to GLP1 secretory responses. Nutrition 29, 813-820.

32. Holst JJ \& McGill MA (2012) Potential new approaches to modifying intestinal GLP-1 secretion in patients with type 2 diabetes mellitus: focus on bile acid sequestrants. Clin Drug Investig 32, 1-14.
33. Egerod KL, Engelstoft MS, Grunddal KV, et al. (2012) A major lineage of enteroendocrine cells coexpress CCK, secretin, GIP, GLP-1, PYY, and neurotensin but not somatostatin. Endocrinology 153, 5782-5795.

34. Kaji I, Karaki S \& Kuwahara A (2014) Short-chain fatty acid receptor and its contribution to glucagon-like peptide-1 release. Digestion 89, 31-36.

35. Freeland KR \& Wolever TMS (2010) Acute effects of intravenous and rectal acetate on glucagon-like peptide-1, peptide $\mathrm{YY}$, ghrelin, adiponectin and tumour necrosis factor- $\alpha$. BrJ Nutr 103, 460-466.

36. Orskov C, Hartmann B, Poulsen SS, et al. (2005) GLP-2 stimulates colonic growth via KGF, released by subepithelial myofibroblasts with GLP-2 receptors. Regul Pept 124, 105-112.

37. Cani PD, Amar J, Iglesias MA, et al. (2007) Metabolic endotoxemia initiates obesity and insulin resistance. Diabetes $\mathbf{5 6}$, 1761-1772.

38. Everard A \& Cani PD (2013) Diabetes, obesity and gut microbiota. Best Pract Res Clin Gastroenterol 27, 73-83.

39. Cani PD (2012) Crosstalk between the gut microbiota and the endocannabinoid system: impact on the gut barrier function and the adipose tissue. Clin Microbiol Infect 18, $50-53$. 